\title{
3D Analysis of Metal Particle Flow Law in Forging Process of Turbine Blade
}

\author{
Fengshou Zhang, YouFu Ma, Binbin. Bao \\ School of Mechanical and Electrical Engineering, Henan University of Science and Technology, \\ Luoyang, 471003, China \\ fengshouzhang@163.com, mayoufu99@163.com, 1293633452@qq.com
}

Keywords: turbine blade; flow law; particle displacement; numerical simulation.

Abstract: The metal particles flow law of the turbine forging process is very hard got to know, due to the complex shape and large deformation of this process. For this situation, a 3D finite element mold was build for this forging process with DEFORM-3D. The results shows that the metal particle flow law in three dimensions is very different in different forging stage and different cross-section of the blade body, the metal particles close to top and bottom die have a smaller displacement than particles in the middle of the cross-section. The particles which close to tenon and shroud have a smaller transverse displacement than particles in the middle blade body. And the particles close to exhaust edge appeared the phenomenon of backflow.

Introduction: The forging process of turbine blade is a typical non steady plastic deformation process, With the features of big flakiness ratio, big torsion angle and big deformation resistance, the flow of metal particles in this process is very complex, the stress and deformation condition are different from parts to parts in this forging process ${ }^{[1-2]}$. Because reasonable metal flow can improve the internal microstructure distribution of the blade, making the inner part of blade formed in accordance with the requirements of the use of the metal flow line ${ }^{[3]}$. To meet the requirements of blade precision forging, it is necessary to analyze the metal flow behaviors in this process. With the organic combination of finite element theory and computer technology making the complex deformation can be accurately prediction, which making predict the deformation condition more efficiently and intuitively ${ }^{[4]}$.

\section{The theoretical basis and the establishment of the finite element model}

The theoretical basis of the finite element mold

Since the plastic deformation of turbine blade forging process is far more than the elastic deformation, usually the plastic deformation was neglected and the forming process was regarded as rigid plastic mode ${ }^{[5]}$. In rigid plastic finite element method, every calculation of each step is based on the geometrical shape and the hardening state of previous step, instead of deriving from the stress increment. So, each of the variable information of rigid plastic deformation can be calculated out accurately ${ }^{[6]}$. In order to be able to analysis the large plastic deformation of the turbine blade forging process, the finite element analysis software DEFORM-3D was adopted, which is an ideal analysis software for numerical simulation of large plastic forming problems ${ }^{[7]}$.

The numerical model and research method.

The displacement of metal particles in forging process occurs simultaneously in three-dimensional space. To grasp the flow law of metal particles in the three-dimensional space, an analysis was given about the flow law of metal particles in different parts of the blade body and different position of the typical cross-sections.

In the forging process of turbine blade, the deformation of the blade body is larger than other parts, and requires the highest precision. While the tenon and shroud requires a lower precision than blade body. So, the research of the blade forging process was focused on the deforming of blade body. Here, three typical cross-sections in indifferent parts of blade body were select out to give a comparative analysis. The locations of the three typical cross-sections are shown in the Fig.1. The cross-section $\mathrm{a}-\mathrm{a}$ close to the tenon, $\mathrm{b}-\mathrm{b}$ is located in the middle of the blade body, and the cross-section c-c close to the shroud. 


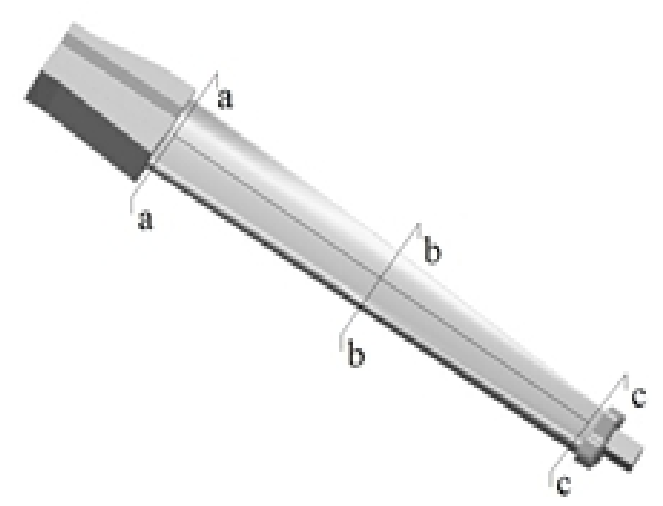

Fig.1. The distribution of typical cross-section on blade body

To study the metal flow law in different cross-sections, five metal particles in the same position was select out from each cross-section. The five metal particles are located in the positions of close to the leading edge, exhaust edge, top die, bottom die and the center of each cross-section. The distributions of metal particles are shown in fig 2. But the twist angle are different in each cross-section, enable to comparative the flow law of the particles, a coordinate transformation was performed to the three cross-sections. The coordinate transformation of cross-section $a-a, b-b, c-c$ were shown in Fig.2.

Enable to calculate the displacement of particles in three directions, use $\Delta X_{\text {in }}, \Delta Y_{\text {in }}, \Delta Z_{\text {in }}$ (i=a,b,c) as the displacement of particles in different position of cross-section a-a, b-b, c-c. $\Delta X_{i n}=X_{i n}-X_{i n 0}, \Delta Y_{a n}=Y_{i n}-Y_{i n 0}, \Delta Z_{a n}=Z_{i n}-Z_{i n 0} ; X_{i n 0}, Y_{i n 0}, Z_{i n 0}$ express the initial coordinate of the particles, and $X_{i n}, Y_{i n}, Z_{i n}$ are the instantaneous coordinate in forging process.

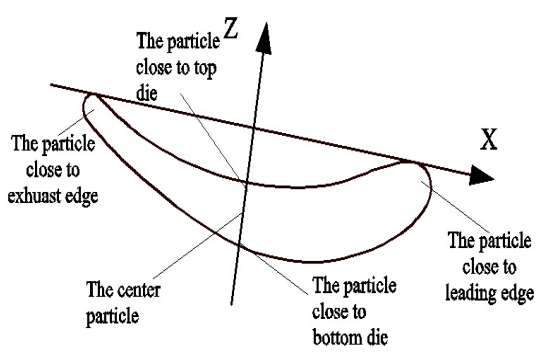

Cross-section a-a

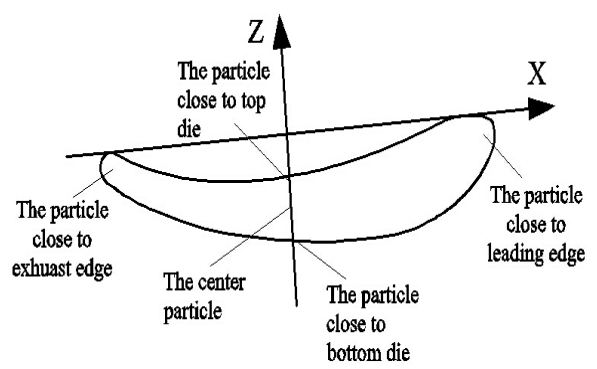

Cross-section b-b

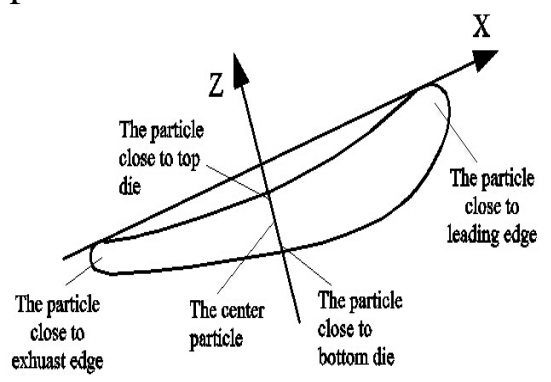

Cross-section c-c

Fig.2. The coordinate transformation of cross-section $a-a, b-b, c-c$

The parameters of the numerical simulation

The material of the material is $1 \mathrm{Cr} 12 \mathrm{NiW} 1 \mathrm{Mo} 1 \mathrm{~V}$, and the initial forging temperature is $1140^{\circ} \mathrm{C}$. To make the numerical simulation accurately and efficiently, the finite element mesh was divided in the absolute method, the mesh size ratio was set as 2 , and the total number of the forging mesh was 68635 . The pressing speed of the top die was $20 \mathrm{~mm} / \mathrm{s}$, and the preheating temperature was $300{ }^{\circ} \mathrm{C}$.

\section{The results and analysis of the numerical simulation}

The three dimensional displacement analysis of particles in different cross-section 


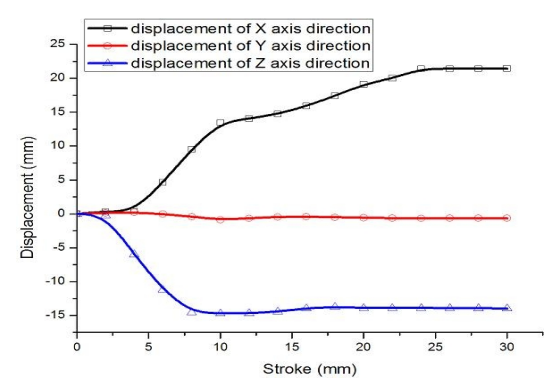

(a)

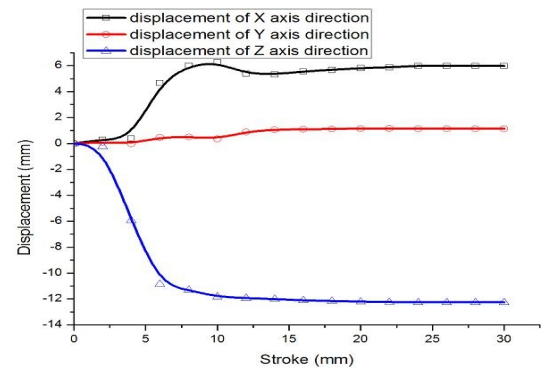

(d)

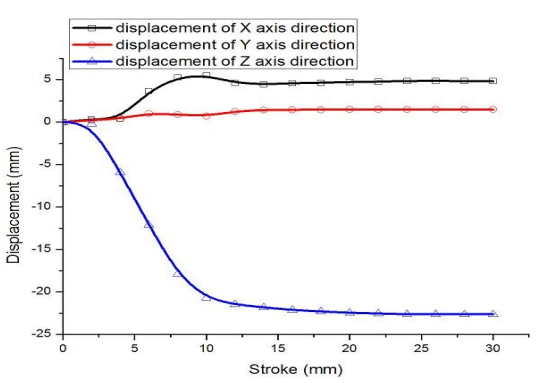

(b)

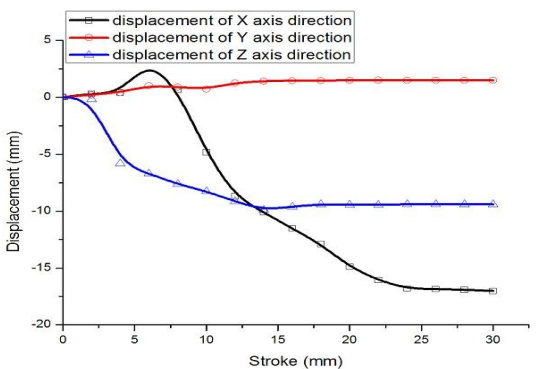

(e)

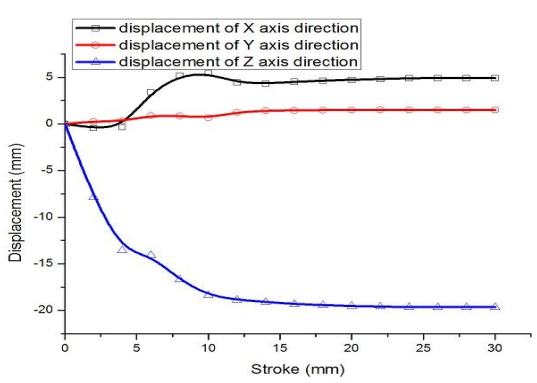

(c)

Fig.3. The metal particles displacement curve of cross-section a-a close to tenon

As shown in Fig.3, the metal particles flow centered on the $\mathrm{X}$ and $\mathrm{Z}$ direction, that is to say the displacement of metal particles mainly happens on the direction of die stroke and the transverse direction of blade body. According to the particles' displacement curve in X direction, it can be seen that the particles which local in the middle of the blade and particle close to exhaust edge flow to positive direction of $\mathrm{X}$ axis first, then flow to the negative direction of $\mathrm{X}$ axis, that is these particles flow to leading edge first, then flow to exhaust edge. It indicates that the flow resistance in exhaust edge bigger than leading edge in the early stage of forging process. With the continue of the forging process, the contact area of dies and pre-forging become enlarge as well as the flow resistance in leading edge, the metal particles begin flow back to the exhaust edge. Due to the lower flow resistance, the particles which local in the leading edge with no black flow phenomenon. But the metal particles have a small displacement in $\mathrm{Y}$ direction owing to the metal distribution of pre-forging is in accordance with the final forging. The particles closer to top die have a bigger displacement in $\mathrm{Z}$ direction, and both flow to the negative direction of $\mathrm{Z}$ axis.

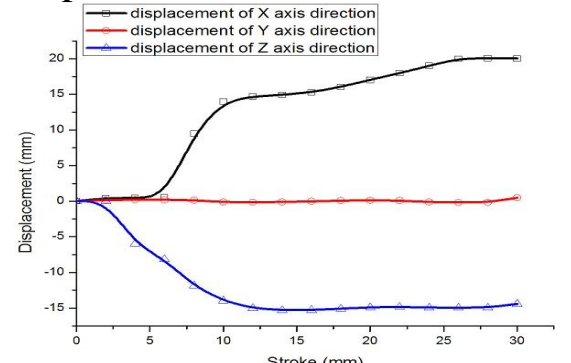

(a)

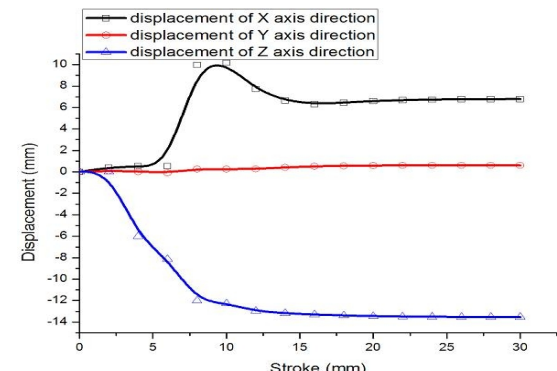

(d)

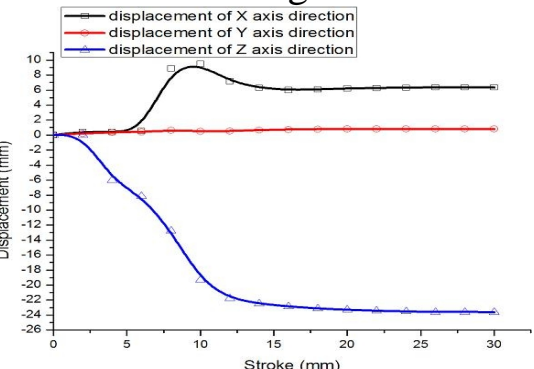

(b)

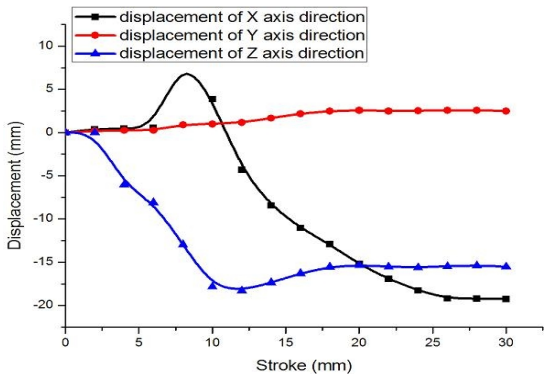

(e)

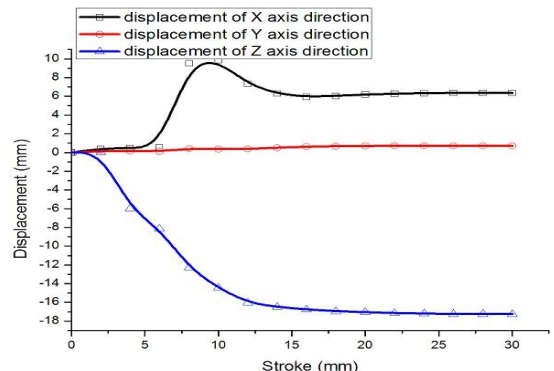

(c)

Fig.4. The metal particles displacement curve of cross-section b-b in the middle of blade body 
After compared with the particles' displacement curve of the typical cross-sections, as shown in the Fig.3, Fig.4 and Fig.5, this regular can be conclude, in the initial phase of the forging process, the particles that in middle of the blade have a larger displacement to the exhaust edge than particles in the cross-sections which close to tenon and shroud. It is because the tenon and shroud hindered the metal flowing that close to them. Hence the particles' displacements of the cross-sections close to tenon and shroud smaller than the middle cross-section of the blade body.

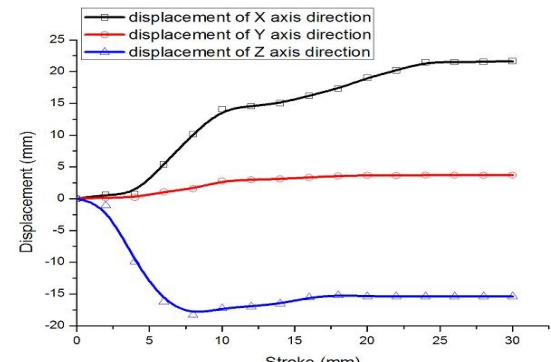

(a)

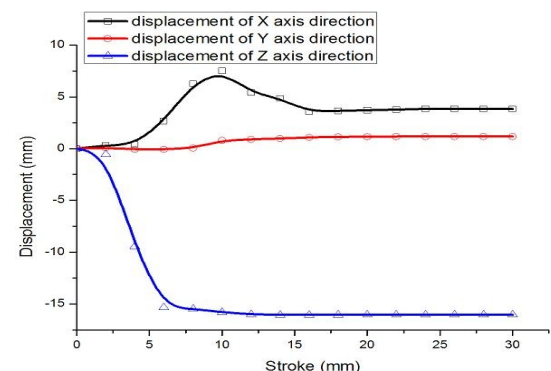

(d)

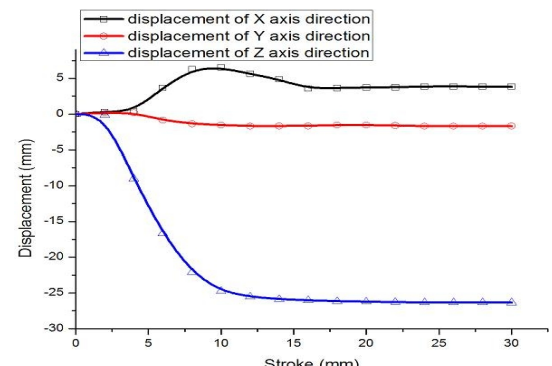

(b)

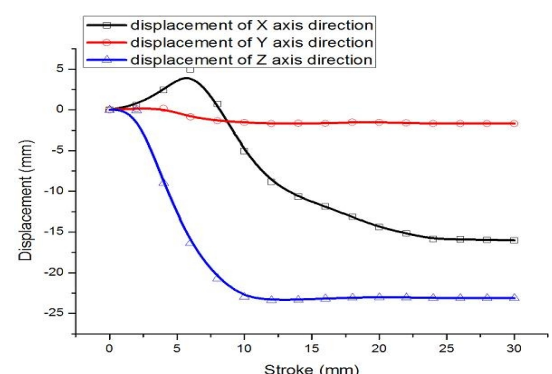

(e)

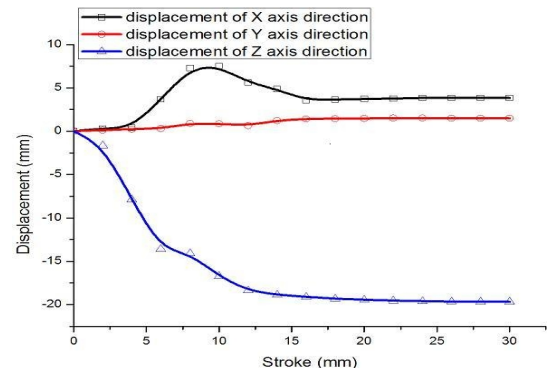

(c)

Fig.5. The metal particles displacement curve of cross-section c-c close to shroud

The analysis of the typical cross-section mesh

In order to directly reflect the situation of mental flowing inside the forging process, the mesh of the typical section were generated based on the mesh generation function of DEFORM-3D. Tracing the mesh of different press amount about upper die, respectively got the mesh deforming figures, which press amount was 15 mm, 20 mm, 25 mm. As shown in the Fig.6.
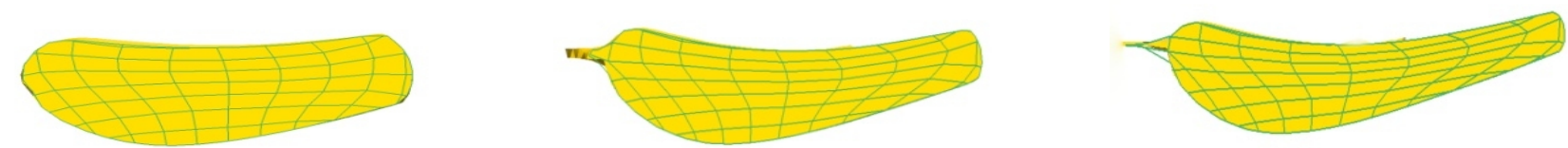

Fig.6. The grid of typical cross-section in different stroke

The velocity field of the blade body

In the process of forging, the longitudinal metal grids of the cross-section sharply bend to the leading edge and exhaust edge with the increasing of the forging stroke. And the grids close to the exhaust edge were more bent than the grids than leading edge. While the transverse mental mesh mainly in tensile deformation on horizontal, the mesh close to the top and bottom mold bends gently with the die's cavity outline.
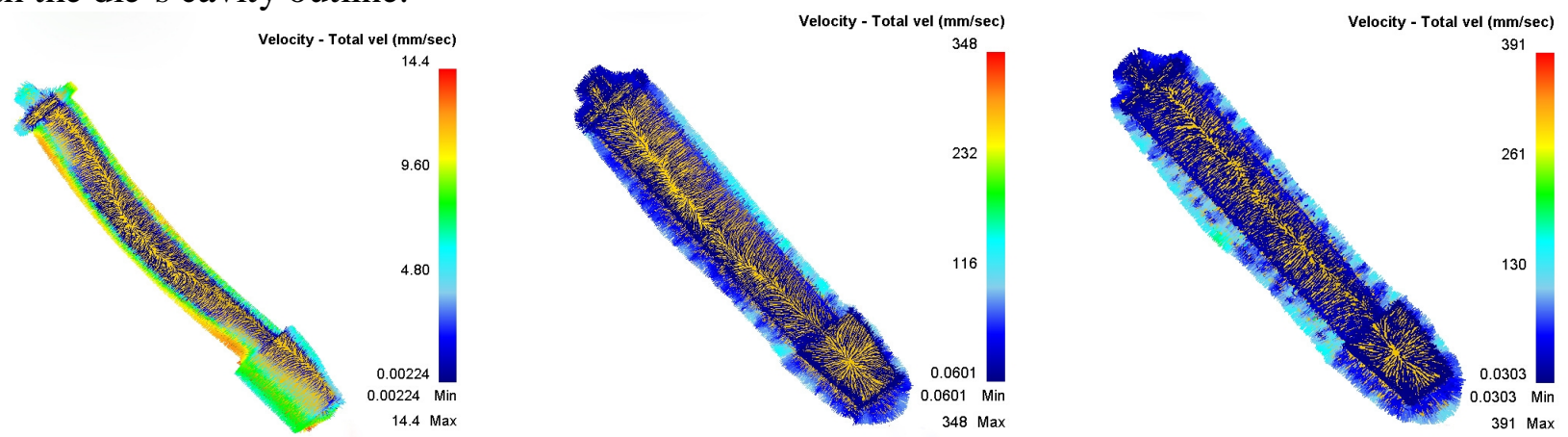

Fig.7. The velocity filed distribution in different stroke 
As shown in Fig.7, it is the velocity field of the blade under the press amount of $15 \mathrm{~mm}, 20 \mathrm{~mm}$ and $25 \mathrm{~mm}$. According to the Figure 7, in the forging process, the metal flow of the blade body appeared diversion phenomenon. From the contact area to the top and bottom die, the metal of the forging respectively flows to the leading edge and exhaust edge. This is because in the initial stage of the forging process, the cross-section of the die cavity close to the leading edge is thicker than the exhaust edge, the flow resistance of the die cavity close to the leading edge is relatively small. Due to more metal material flows to the cavity close to leading edge. Thus the diversion phenomenon occurs first in the region near the exhaust edge. Because of the hinder of the shroud and tenon, the diversion phenomenon near to the shroud and tenon part slightly away from the exhaust edge. With the die cavity of the leading edge is gradually filled with material, the metal begin flow to the gutter of the leading edge, the resistance of the die cavity of leading edge is gradually increased, then metal starts to flow back. The boundary of diversion gradually moved to the exhaust edge. In the final stage of the forging process, the cavity of leading and exhaust edge have been filled, the metal flow resistances of both edge are equal, so the boundary of diversion moved to the middle part of the blade body.

\section{Conclusions}

(1) The metal particle's flow behavior numerical model in the process of the turbine blade's forging forming was established, and the flow law of the metal particles was accurately simulated and analyzed. This provides a practical model for the internal metal particle flow analysis during the blade forging forming process.

(2) In the process of turbine blade forging forming, the size of the internal metal particle's displacement is largely influenced by its location at the blade body; meanwhile the metal particle's position in the cross section has a great effect on its flow trend.

\section{Acknowledgements:}

This subject is supported by National Natural Science Foundation of China, (51475146).

Bibliography: Fengshou Zhang, (1972- ), male, the Han nationality, natively place: Henan, Professor, Ph.D., research area: Advanced Manufacturing Technology.

Corresponding Author: Youfu Ma, E-mail: mayoufu99@163.com.

\section{References}

[1]. L. Li, G.X. Qi and L.K. Shi: 'Simulation of TC4 Titanium Alloy Blades Final Forging Forming', Journal of Shenyang Ligong University, 2014, vol 33, NO.2, 21-25.

[2]. J. Zhang: 'Metal Flow Behavior in Complex Stress Field', PhD thesis, Harbin Institute of Technology, Harbin, China, 2008, 11-35.

[3]. J. Wang: 'The Impact of The Pre-forging Shape on Metal Flow During Forging Process of High Pressure Guide Blade', China Metal Forming Equipment \& Manufacturing Technology, 2014, vol 49, NO.3, 60-62.

[4]. G.X. Zhang: 'RL1016R Blade's Die Forging shaping and Its Finite Element Analysis', Casting Forging Welding, 2012, vol 41, NO.01, 106-110.

[5]. Y. Shao, P.Y. Guo: 'Optimal Pre-forging Design of Blade Body Based on Deform Software', Forming \& Stamping Technology, 2012, vol 37, NO.5, 12-16.

[6]. K. Xu, R. Shao: 'Study on Blade Forging Die Design Based on Deform-3D', Hot Working Technology, 2014, vol 43, NO.5, 148-153.

[7]. Z.T. Wang, X.L. Duan, H.Y. Zhang, etal: '3D FEM simulation of multi-pass upsetting and stretching for T 11 alloy’, Forming \& Stamping Technology, 2012, vol 34, NO.1, 1-5. 